\title{
PERFIL DE ÁCIDOS GRAXOS TRANS DE ÓLEO E GORDURA HIDROGENADA DE SOJA NO PROCESSO DE FRITURA ${ }^{1}$
}

\author{
Elaine Abrão Assef SANIBAL ${ }^{2, *}$, Jorge MANCINI FILHO ${ }^{2}$
}

\section{RESUMO}

O objetivo desse estudo foi avaliar as alterações dos ácidos graxos e a formação de isômeros trans, durante o aquecimento de óleo de soja (OS) e gordura parcialmente hidrogenada de soja (GPHS) no processo de fritura de batata por 100 horas com reposição lipídica. O perfil de ácidos graxos foi avaliado através de cromatografia gasosa em coluna capilar de $100 \mathrm{~m}$. Os ácidos graxos monoinsaturados trans foram os predominantes entre os isômeros trans. A partir de 10 horas de fritura, o OS formou 2,1\% de isômeros mono trans e ao final de 50 horas este valor passou a $14,3 \%$ contra uma diminuição do total de ácidos graxos poliinsaturados, que passou de $59,9 \%$ antes do processamento para 32,6\% após 50h de fritura. Entretanto, a GPHS apresentou 20,2\% de ácidos graxos mono trans antes de ser submetida à fritura e após 50 horas apresentou uma concentração de $28 \%$. Houve também, uma diminuição do total de ácidos graxos essenciais séries $\omega 6$ e $\omega 3$, de $12,8 \%$ para $7,3 \%$ no mesmo período. Os resultados obtidos revelaram que isômeros trans são formados no óleo e na gordura durante o processo de fritura, sendo que a formação de isômeros trans, ocorreu em menor proporção na GPHS, confirmando a sua maior estabilidade em relação ao OS. Estes resultados indicam a importância de se identificar os ácidos graxos trans nos óleos e gorduras ulizadas em processos de fritura.

Palavras-chave: lipídios; fritura; ácidos graxos; isômeros trans.
\end{abstract}

\section{SUMMARY}

FATTY ACIDS TRANS PROFILE OF OIL AND HIDROGENATED SOY FAT IN FRYING PROCESS. The objective of this study, was to examine the alterations of the fatty acids profile and the formation of trans fatty acids isomers during heating of soy oil (SO) and partially hydrogenated soy fat (PHSF) in the process of french frying. The fatty acids profile was examined by gas chromatography with a $100 \mathrm{~m}$ capillary column. The fatty acids trans monounsaturated were the predominant among trans isomers. The isomers trans monounsaturated corresponded to $2.1 \%$ of the total, after 10 hours of frying, and $14.3 \%$ after $50 \mathrm{~h}-$ frying. Poliunsaturated fatty acids decreased from $59.9 \%$ to $32.6 \%$ after $50 \mathrm{~h}$ - frying. For the PHSF, the initial content of $20.2 \%$ of trans monounsaturated isomers increased to $28 \%$ after $50 \mathrm{~h}$ - frying, and $\omega_{3}$ and $\omega_{6}$ - essential fatty acids decreased from 12.8 to $7.3 \%$, in the same period. These results confirmed the formation of trans isomers in oil and hydrogenated oil in frying process, and that the trans isomers formation occurred in lower proportion in the hydrogenated oil. These results show the importance of identifying the trans fatty acids in oils and fats utilized in frying process.

Keywords: lipids; frying; fatty acids; trans isomers.

\section{1 - INTRODUÇÃO}

A complexidade do processo de fritura induz diferentes reações químicas, as quais resultam na formação de diversos produtos da decomposição de óleos e gorduras $[8,9]$. Suas implicações sensoriais e nutricionais são as principais preocupações quanto aos alimentos fritos $[2,11]$.

Os compostos formados pela decomposição de ácidos graxos insaturados durante o processo de fritura afetam a disponibilidade dos ácidos graxos essenciais, linoléico e $\alpha$-linolênico [5, 16], responsáveis pela biossíntese dos ácidos araquidônico, eicosapentaenóico e docosahexaenóico, na formação das prostaglandinas, tromboxanos e prostaciclinas, compostos que participam da regulação da pressão arterial, freqüência cardíaca, resposta imunológica, dos processos da coagulação sangüínea e do funcionamento do sistema nervoso central [7].

Gorduras utilizadas em fritura são fontes de ácidos graxos trans na dieta. OVESEN, LETH \& HANSEN [10],

\footnotetext{
1. Recebido para publicação em 09/10/2001. Aceito para publicação em 24/09/2003 (000744).

2. Universidade de São Paulo - Depto. de Alimentos e Nutrição Experimental Cx. Postal 66083, CEP 05315-970 S.P. sanibal@usp.br

*A quem a correspondência deve ser enviada.
}

avaliaram o perfil de ácidos graxos das gorduras utilizadas pelas redes de fast-food, Burger King e Mc Donald's. As gorduras utilizadas por ambas as redes de fast-food continham altas concentrações de ácidos graxos monoinsaturados trans, principalmente, o ácido elaídico (C18:1t), $21,9 \pm 2,9 \%$ e $16,6 \pm 0,4 \%$, respectivamente. No entanto, não há um consenso se os alimentos fritos podem ser considerados uma fonte importante de ácidos graxos trans. ROMERO, CUESTA \& SANCHES-MUNIZ [13], consideram que os ácidos graxos trans são constituintes em quantidades irrelevantes nos alimentos fritos.

Os isômeros geométricos trans de ácidos graxos insaturados são formados no processo de fritura, assim como no refino de óleos e no processo de hidrogenação, por mecanismo induzido termicamente [14]). São identificados em vários tipos de alimentos, como por exemplo: em margarinas vegetais, massas e recheios de biscoitos, nas formulações de bases para sopas e cremes, nos produtos de panificação, nas coberturas para adesão de especiarias e açúcares, entre outros. Pelas características estruturais os ácidos graxos na forma trans têm seu ponto de fusão mais elevado, quando comparado com seu isômero cis correspondente, e próximo ao ponto de fusão do ácido graxo saturado com mesmo número de átomos de carbono. Sendo assim, os isômeros trans podem ser considerados como um intermediário entre um ácido graxo original insaturado 
cis e um ácido graxo completamente saturado. Os ácidos graxos trans de maior ocorrência são os monoinsaturados, mas vários isômeros diinsaturados, ou mesmo, triinsaturados podem ser formados a partir dos ácidos linoléico e linolênico [17].

Há controvérsias sobre o significado dos ácidos graxos trans na nutrição humana, particularmente no que concerne seus efeitos negativos no perfil das lipoproteínas, com implicações desfavoráveis na aterosclerose. Há algumas evidências de que modesta ingestão de ácidos graxos trans pode afetar o perfil das lipoproteínas, aumentando a lipoproteína de baixa densidade (LDL), diminuindo a lipoproteína de alta densidade (HDL) e aumentando a lipoproteína a (Lpa). No entanto, estas observações têm sido discutidas, procurando avaliar se os ácidos graxos trans são melhores ou piores do que os ácidos graxos saturados, quando utilizados na produção de alguns alimentos fritos, gorduras e margarinas $[1,6,15]$.

Com o reconhecimento da participação das gorduras saturadas no aumento do colesterol sangüíneo, houve maior interesse na utilização dos óleos hidrogenados na fabricação e processamento dos alimentos, acreditando-se que os ácidos graxos insaturados na forma trans não apresentariam os mesmos efeitos dos ácidos graxos saturados [19].

Apesar das controvérsias, os ácidos graxos trans têm sido cada vez mais pesquisados, tanto no campo tecnológico, como em relação aos aspectos nutricionais. Várias propostas têm surgido como uma alternativa na formulação da gordura vegetal hidrogenada sem isômeros trans. Modificações futuras na legislação, possivelmente incluirão maiores informações para os consumidores. A Food and Drug Administration (FDA) sugeriu, em 1999, que a quantidade de ácidos graxos trans fosse incluída nos rótulos dos produtos alimentícios. Foi recomendado também que, quando somado aos ácidos graxos saturados, fosse informada a quantidade específica de ácidos graxos trans [3].

Com as perspectivas de se obter mais informações quanto à presença dos ácidos graxos trans em lipídios utilizados em processamento, este estudo avaliou o perfil dos ácidos graxos, a formação de isômeros geométricos trans e a diminuição dos ácidos graxos essenciais, durante o aquecimento de óleo e gordura de soja, no processo de fritura de batata palito pré-frita congelada.

\section{2 - MATERIAIS E MÉTODOS}

O experimento de fritura foi conduzido no Laboratório de Lípides da Faculdade de Ciências Farmacêuticas da Universidade de São Paulo. O óleo de soja (OS) da marca Lisa e a gordura parcialmente hidrogenada de soja (GPHS) da marca Frygill, foram cedidos pela empresa Cargill Agrícola S.A., Brasil, São Paulo. As batatas palito pré-fritas congeladas da marca Mc Cain, foram cedidas pela Distribuidora Pratigel - Ind. e Com. de Alimentos Ltda, Brasil, São Paulo.
Para a operação de fritura em laboratório foi utilizada uma fritadeira elétrica com capacidade de $5 \mathrm{~L}$ da marca Fritanella-Walita.

O tempo total de processamento foi de 50 horas com temperatura controlada a $180^{\circ} \mathrm{C} \pm 5^{\circ} \mathrm{C}$, tanto para o OS quanto para a GPHS. O processamento foi conduzido cinco horas por dia, durante dez dias. A cada hora foram realizadas duas frituras com duração de 4 minutos cada, totalizando 100 operações de fritura em 50 horas de processamento. A quantidade de batata a cada fritura, correspondeu a $10 \% \mathrm{p} / \mathrm{v}$ de óleo ou gordura, totalizando no final de 50 horas de fritura, $2310 \mathrm{~g}$ de batatas fritas em cada meio de fritura.

A cada 10 horas foi feita filtragem do OS e da GPHS, com a utilização de papel de filtro e bomba a vácuo. Para análises físicas e químicas do OS e da GPHS, $300 \mathrm{~mL}$ de amostra foram coletados e acondicionados em vidro âmbar com nitrogênio e acondicionado em freezer $\mathrm{a}-18^{\circ} \mathrm{C}$. Em seguida, o volume do meio de fritura foi controlado de acordo com 10\% de reposição em relação ao volume final.

Para a análise do perfil de ácidos graxos as amostras de OS e GPHS foram esterificadas pelo método de HARTMAN \& LAGO [4]. Na identificação e quantificação dos ácidos graxos foi utilizado um cromatógrafo a gás CG 17/A Shimadzu/Class CG 10, com coluna capilar SP 2560 de sílica fundida de 100m de comprimento e $0,25 \mathrm{~mm}$ de diâmetro interno, com detector de ionização de chama, hélio como gás de arraste e fluxo de $1 \mathrm{~mL} /$ minuto. As condições cromatográficas foram as seguintes: temperatura do detector de $275^{\circ} \mathrm{C}$, temperatura do vaporizador de $275^{\circ} \mathrm{C}$, temperatura inicial da coluna de $170^{\circ} \mathrm{C}$ durante 50 minutos, temperatura final da coluna de $225^{\circ} \mathrm{C}$, durante 30 minutos, velocidade de aquecimento $4^{\circ} \mathrm{C}$ por minuto e split $1 / 50$. Foram utilizadas três repetições para a análise do perfil de ácidos graxos. A determinação da área de cada pico foi controlada de acordo com as instruções do fabricante. Foram utilizadas três repetições para a análise do perfil de ácidos graxos $\mathrm{O}$ método para a identificação dos ácidos graxos foi baseado no trabalho de RATNAYAKE et al. [12] e através da utilização de padrões internos Sigma.

As análises da média e desvio padrão foram realizadas pelo Microsoft Excel, versão 5.0 da Microsoft Inc., enquanto que para as análises de variância foi utilizado o teste de Tukey-Kramer de múltiplas comparações, empregando-se o programa GraphPad Instat, versão 2.01 da GraphPad Software. Para os resultados entre as médias foi fixado o nível de erro em $5 \%$.

\section{3 - RESULTADOS E DISCUSSÃO}

De acordo com o perfil de ácidos graxos insaturados, o OS (Tabela 1) no tempo 0 (sem utilização), continha alta concentração em ácido graxo linoléico (C18:2n-6), $55,1 \% \pm 0,05$, concentração intermediária em ácido graxo oléico (C18:1n-9), 21,3\% $\pm 0,08$ e baixa concentração em ácido linolênico, 4,79\% $\pm 0,01$. Enquanto que a GPHS (Tabela 2), continha alta concentração em ácido graxo 
oléico (C18:1n-9), 26,8\% $\pm 1,53$, concentração intermediária em ácido graxo linoléico (C18:2n-6), 11,9\% $\pm 0,01$ e baixa concentração em ácido linolênico (C18:3n-3), $0,4 \% \pm 0,00$. As características iniciais da composição de ácidos graxos do OS (Tabela 1) e da GPHS (Tabela 2) dadas no tempo 0 (sem utilização), mostra que o OS apresentou uma concentração de $47,1 \%$ a mais de ácidos graxos poliinsaturados em relação à GSPH. O perfil de ácidos graxos insaturados, exceto para o ácido oléico (C18:1n-9), tanto no OS como na GPHS, mostrou mudanças sistemáticas durante o processo de fritura (Tabelas 1 e 2). O OS apresentou perda maior na concentração de poliinsaturados em relação à GPHS. No final das 50 horas de fritura, o OS ainda apresentava uma alta concentração de ácidos graxos poliinsaturados (32,6\%), em relação à GSPH (7,3\%) (Tabelas 1 e 2).

TABELA 1. Composição de ácidos graxos (\%) do OS em diversos tempos de utilização no processo de fritura de imersão de batata palito pré-frita congelada.

\begin{tabular}{|c|c|c|c|c|c|c|}
\hline & \multicolumn{6}{|c|}{ Tempo de fritura (horas) } \\
\hline & 0 & 10 & 20 & 30 & 40 & 50 \\
\hline Ácidos Graxos & Média & Média & Média & Média & Média & Média \\
\hline C14:0 & $0,07 \pm 0,00$ & $0,07 \pm 0,01$ & $0,08 \pm 0,00$ & $0,09 \pm 0,00$ & $0,09 \pm 0,01$ & $0,10 \pm 0,00$ \\
\hline C16:0 & $10,84 \pm 0,09$ & $11,38 \pm 0,09$ & $11,62 \pm 0,05$ & $12,23 \pm 0,03$ & $12,53 \pm 0,05$ & $12,37 \pm 0,07$ \\
\hline C16:1 & $0,07 \pm 0,01$ & $0,06 \pm 0,01$ & $0,06 \pm 0,01$ & $0,07 \pm 0,01$ & $0,06 \pm 0,01$ & $0,06 \pm 0,00$ \\
\hline $\mathrm{C} 17: 0$ & $0,08 \pm 0,01$ & $0,08 \pm 0,01$ & $0,08 \pm 0,01$ & $0,09 \pm 0,00$ & $0,10 \pm 0,00$ & $0,10 \pm 0,00$ \\
\hline C18:0 & $3,32 \pm 0,02$ & $4,86 \pm 0,05$ & $6,15 \pm 0,02$ & $7,52 \pm 0,04$ & $8,77 \pm 0,11$ & $9,61 \pm 0,10$ \\
\hline C18:1 9t & $0,00 \pm 0,00$ & $0,76 \pm 0,03$ & $1,32 \pm 0,08$ & $1,78 \pm 0,03$ & $2,45 \pm 0,39$ & $2,84 \pm 0,30$ \\
\hline C18:1 trans & $0,00 \pm 0,00$ & $1,55 \pm 0,72$ & $5,03 \pm 0,14$ & $7,33 \pm 0,20$ & $9,54 \pm 0,37$ & $11,43 \pm 0,47$ \\
\hline C18:1 9c & $21,25 \pm 0,08$ & $21,83 \pm 0,18$ & $21,98 \pm 0,16$ & $22,57 \pm 0,19$ & $22,62 \pm 0,12$ & $22,22 \pm 0,10$ \\
\hline C18:1 10c & $1,37 \pm 0,01$ & $1,50 \pm 0,01$ & $1,60 \pm 0,00$ & $1,72 \pm 0,01$ & $1,82 \pm 0,02$ & $1,87 \pm 0,01$ \\
\hline C18:1 11c & - & $0,37 \pm 0,03$ & $0,72 \pm 0,00$ & $1,05 \pm 0,01$ & $1,33 \pm 0,01$ & $1,47 \pm 0,01$ \\
\hline $\mathrm{C} 18: 1 \mathrm{12c}$ & - & $0,13 \pm 0,00$ & $0,20 \pm 0,01$ & $0,26 \pm 0,00$ & $0,33 \pm 0,00$ & $0,39 \pm 0,00$ \\
\hline $\mathrm{C} 18: 113 \mathrm{c}$ & - & - & $0,19 \pm 0,01$ & $0,28 \pm 0,01$ & $0,38 \pm 0,01$ & $0,47 \pm 0,00$ \\
\hline C18:1 14c & - & - & - & - & 0,13 & $0,16 \pm 0,01$ \\
\hline $\mathrm{C} 18: 29 \mathrm{t}, 12 \mathrm{c}$ & - & - & $0,09 \pm 0,00$ & $0,12 \pm 0,00$ & $0,14 \pm 0,01$ & $0,15 \pm 0,01$ \\
\hline$C 18: 29 t, 12 t$ & - & - & $0,16 \pm 0,00$ & $0,24 \pm 0,01$ & $0,30 \pm 0,01$ & $0,34 \pm 0,00$ \\
\hline$C 18: 29 c, 12 t$ & $0,57 \pm 0,01$ & $0,81 \pm 0,01$ & $0,66 \pm 0,25$ & $0,78 \pm 0,00$ & $0,77 \pm 0,01$ & $0,74 \pm 0,02$ \\
\hline $\mathrm{C} 18: 29 \mathrm{t}, 12 \mathrm{c}$ & $0,15 \pm 0,03$ & $0,77 \pm 0,01$ & $0,65 \pm 0,19$ & $0,39 \pm 0,04$ & $0,70 \pm 0,01$ & $0,67 \pm 0,01$ \\
\hline C18:2 trans & - & - & - & $0,34 \pm 0,04$ & - & - \\
\hline C18:2 9c,12c & $55,11 \pm 0,05$ & $49,36 \pm 0,37$ & $43,65 \pm 0,20$ & $38,08 \pm 0,02$ & $33,56 \pm 0,14$ & $30,69 \pm 0,18$ \\
\hline$C 18: 29 c, 15 c$ & - & - & - & - & - & $0,05 \pm 0,01$ \\
\hline $\mathrm{C} 20: 0$ & $0,35 \pm 0,01$ & $0,37 \pm 0,01$ & $0,38 \pm 0,00$ & $0,41 \pm 0,00$ & $0,43 \pm 0,01$ & $0,42 \pm 0,00$ \\
\hline C18:3 trans & $1,38 \pm 0,03$ & $1,68 \pm 0,01$ & $1,52 \pm 0,01$ & $1,24 \pm 0,01$ & $1,06 \pm 0,00$ & $0,95 \pm 0,01$ \\
\hline $\mathrm{C} 18 \mathrm{~s} 3 \mathrm{9c}, 12 \mathrm{c}, 15 \mathrm{c}$ & $4,79 \pm 0,01$ & $3,59 \pm 0,03$ & $2,96 \pm 0,01$ & $2,37 \pm 0,01$ & $1,98 \pm 0,01$ & $1,84 \pm 0,02$ \\
\hline C22:0 & $0,43 \pm 0,01$ & $0,46 \pm 0,00$ & $0,47 \pm 0,00$ & $0,50 \pm 0,01$ & $0,51 \pm 0,01$ & $0,50 \pm 0,01$ \\
\hline C24:0 & $0,15 \pm 0,01$ & $0,15 \pm 0,01$ & $0,16 \pm 0,00$ & $0,17 \pm 0,00$ & $0,16 \pm 0,01$ & $0,16 \pm 0,01$ \\
\hline Não ident & 0,07 & 0,22 & 0,27 & 0,37 & 0,24 & 0,40 \\
\hline Total saturados & $15,24^{\dagger}$ & $17,38^{\mathrm{e}}$ & $18,94^{\mathrm{d}}$ & $21,01^{\mathrm{c}}$ & $22,59^{b}$ & $23,26^{\mathrm{a}}$ \\
\hline Total & $22,69^{e}$ & $23,89^{d}$ & $24,75^{c}$ & $25,95^{\mathrm{b}}$ & $26,67^{\mathrm{a}}$ & $26,64^{a}$ \\
\hline Total polins. & $59,90^{\mathrm{a}}$ & $52,95^{\mathrm{b}}$ & $46,61^{c}$ & $40,45^{d}$ & $35,54^{e}$ & $32,58^{f}$ \\
\hline Total mono trans & $0,00^{f}$ & $2,10^{\mathrm{e}}$ & $6,35^{\mathrm{d}}$ & $9,11^{\mathrm{c}}$ & $11,99^{\mathrm{b}}$ & $14,27^{\mathrm{a}}$ \\
\hline Total poli trans & $2,10^{\mathrm{e}}$ & $3,26^{\mathrm{a}}$ & $3,08^{\mathrm{b}}$ & $3,11^{\mathrm{a}}$ & $2,97^{\mathrm{c}}$ & $2,85^{\mathrm{d}}$ \\
\hline
\end{tabular}

a,b.c... Médias na mesma linha seguidas de letras diferentes, diferem significativamente entre si ao nível de $5 \%$.

As médias das concentrações do total de ácidos graxos saturados no OS (Tabela 1) nos tempos 0,30 e 50 horas de fritura foram: $15,2 \%, 21,0 \%$ e $23,3 \%$ e na GPHS (Tabela 2) foram: $21,9 \%, 24,0 \%$ e 25,6\%. O aumento observado dos ácidos graxos saturados é decorrente da diminuição proporcional dos ácidos graxos poliinsaturados.
TABELA 2. Composição de ácidos graxos (\%) da GPHS em diversos tempos de utilização no processo de fritura de imersão de batata palito pré-frita congelada.

\begin{tabular}{|c|c|c|c|c|c|c|}
\hline & \multicolumn{6}{|c|}{ Tempo de fritura (horas) } \\
\hline & 0 & 10 & 20 & 30 & 40 & 50 \\
\hline Ácidos Graxos & Média & Média & Média & Média & Média & Média \\
\hline C14:0 & $0,19 \pm 0,01$ & $0,19 \pm 0,01$ & $0,18 \pm 0,00$ & $0,17 \pm 0,00$ & $0,18 \pm 0,01$ & $0,18 \pm 0,02$ \\
\hline C16:0 & $13,37 \pm 0,02$ & $13,33 \pm 0,02$ & $13,25 \pm 0,03$ & $13,24 \pm 0,02$ & $13,27 \pm 0,07$ & $13,11 \pm 0,17$ \\
\hline C16:1 & $0,11 \pm 0,00$ & $0,10 \pm 0,00$ & $0,10 \pm 0,01$ & $0,09 \pm 0,00$ & $0,08 \pm 0,01$ & $0,08 \pm 0,02$ \\
\hline C17:0 & $0,08 \pm 0,01$ & $0,09 \pm 0,01$ & $0,09 \pm 0,00$ & $0,09 \pm 0,00$ & $0,10 \pm 0,01$ & $0,10 \pm 0,01$ \\
\hline C18:0 & $7,34 \pm 0,05$ & $8,53 \pm 0,01$ & $9,44 \pm 0,04$ & $9,50 \pm 1,21$ & $10,86 \pm 0,03$ & $11,17 \pm 0,33$ \\
\hline C18:1 9t & $8,31 \pm 0,97$ & $9,58 \pm 0,27$ & $10,63 \pm 0,66$ & $11,18 \pm 0,12$ & 2 $11,66 \pm 0,16$ & $12,37 \pm 1,15$ \\
\hline C18:1 trans & $11,91 \pm 1,96$ & $14,02 \pm 0,20$ & $14,30 \pm 0,73$ & $15,88 \pm 1,48$ & $15,46 \pm 0,26$ & $15,64 \pm 1,19$ \\
\hline C18:1 9c & $26,82 \pm 1,53$ & $24,64 \pm 0,03$ & $24,29 \pm 0,06$ & $23,81 \pm 0,46$ & $23,59 \pm 0,11$ & $23,67 \pm 0,48$ \\
\hline C18:1 10c & $1,92 \pm 0,03$ & $2,02 \pm 0,00$ & $2,05 \pm 0,02$ & $2,09 \pm 0,03$ & $2,13 \pm 0,01$ & $2,20 \pm 0,00$ \\
\hline C18:1 11c & $6,07 \pm 0,00$ & $5,88 \pm 0,01$ & $5,76 \pm 0,03$ & $5,66 \pm 0,03$ & $5,51 \pm 0,03$ & $5,41 \pm 0,05$ \\
\hline C18:1 12c & $0,31 \pm 0,00$ & $0,37 \pm 0,00$ & $0,42 \pm 0,00$ & $0,46 \pm 0,01$ & $0,49 \pm 0,00$ & $0,52 \pm 0,01$ \\
\hline C18:1 13c & $0,36 \pm 0,00$ & $0,44 \pm 0,02$ & $0,51 \pm 0,01$ & $0,55 \pm 0,01$ & $0,60 \pm 0,01$ & $0,62 \pm 0,02$ \\
\hline C18:1 14c & $0,20 \pm 0,00$ & $0,20 \pm 0,00$ & $0,20 \pm 0,00$ & $0,20 \pm 0,01$ & $0,21 \pm 0,01$ & $0,21 \pm 0,02$ \\
\hline C18:1 15c & $0,22 \pm 0,01$ & $0,26 \pm 0,01$ & $0,27 \pm 0,01$ & $0,29 \pm 0,00$ & $0,29 \pm 0,01$ & $0,31 \pm 0,01$ \\
\hline C18:2 9t,12c/9c, 12 & $0,80 \pm 0,01$ & $0,72 \pm 0,01$ & $0,67 \pm 0,01$ & $0,62 \pm 0,00$ & $0,59 \pm 0,00$ & $0,57 \pm 0,00$ \\
\hline C18:2 9t,12t & $1,78 \pm 0,01$ & $1,68 \pm 0,01$ & $1,61 \pm 0,01$ & $1,54 \pm 0,01$ & $1,50 \pm 0,01$ & $1,46 \pm 0,01$ \\
\hline C18:2 9c, 12t & $2,71 \pm 0,02$ & $2,43 \pm 0,00$ & $2,20 \pm 0,01$ & $1,99 \pm 0,01$ & $1,84 \pm 0,01$ & $1,69 \pm 0,03$ \\
\hline C18:2 9t, 12c & $2,43 \pm 0,07$ & $2,11 \pm 0,02$ & $1,89 \pm 0,04$ & $1,74 \pm 0,07$ & $1,55 \pm 0,02$ & $1,43 \pm 0,03$ \\
\hline C18:2 trans & $0,84 \pm 0,04$ & $0,91 \pm 0,01$ & $0,84 \pm 0,09$ & $0,74 \pm 0,01$ & $0,74 \pm 0,02$ & $0,74 \pm 0,02$ \\
\hline C18:2 9c,12c & $11,89 \pm 0,01$ & $10,43 \pm 0,02$ & $9,27 \pm 0,04$ & $8,23 \pm 0,05$ & $7,40 \pm 0,06$ & $6,72 \pm 0,03$ \\
\hline C18:2 9c,15c & $0,45 \pm 0,02$ & $0,43 \pm 0,01$ & $0,39 \pm 0,01$ & $0,37 \pm 0,01$ & $0,36 \pm 0,00$ & $0,34 \pm 0,02$ \\
\hline $\mathrm{C} 20: 0$ & $0,37 \pm 0,01$ & $0,38 \pm 0,00$ & $0,39 \pm 0,00$ & $0,40 \pm 0,00$ & $0,41 \pm 0,00$ & $0,40 \pm 0,01$ \\
\hline C18:3 trans & $0,14 \pm 0,02$ & $0,11 \pm 0,01$ & $0,08 \pm 0,03$ & $0,05 \pm 0,00$ & - & - \\
\hline C18:3 9c,12c,15c & $0,42 \pm 0,00$ & $0,37 \pm 0,03$ & $0,30 \pm 0,01$ & $0,27 \pm 0,01$ & $0,30 \pm 0,08$ & $0,24 \pm 0,01$ \\
\hline C22:0 & $0,40 \pm 0,00$ & $0,41 \pm 0,00$ & $0,42 \pm 0,01$ & $0,43 \pm 0,00$ & $0,44 \pm 0,00$ & $0,44 \pm 0,02$ \\
\hline C24:0 & $0,14 \pm 0,00$ & $0,14 \pm 0,01$ & $0,14 \pm 0,00$ & $0,15 \pm 0,00$ & $0,15 \pm 0,00$ & $0,15 \pm 0,00$ \\
\hline Não identificados & 0,42 & 0,23 & 0,31 & 0,26 & 0,29 & 0,23 \\
\hline Total saturados & $21,8 b 9^{d}$ & $23,07^{\text {cd }}$ & $23,91^{\mathrm{c}}$ & $23,98^{\mathrm{bc}}$ & $25,41^{a b}$ & $25,55^{\mathrm{a}}$ \\
\hline Total monoins. & $36,01^{\mathrm{a}}$ & $33,91^{b}$ & $33,60^{b}$ & $33,15^{b}$ & $32,90^{b}$ & $33,02^{b}$ \\
\hline Total polins. & $12,76^{\mathrm{a}}$ & $11,23^{b}$ & $9,96^{\mathrm{c}}$ & $8,87^{d}$ & $8,06^{\mathrm{e}}$ & $7,30^{f}$ \\
\hline Total mono trans & $20,22^{\mathrm{d}}$ & $23,60^{b}$ & $24,93^{b c}$ & $27,06^{a c}$ & $27,12^{a c}$ & $28,01^{a}$ \\
\hline Total poli trans & $8,70^{a}$ & $7,96^{b}$ & $7,29^{c}$ & $6,68^{d}$ & $6,22^{\mathrm{e}}$ & $5,89^{f}$ \\
\hline
\end{tabular}

a,b,c... Médias na mesma linha seguidas de letras diferentes, diferem significativamente entre si ao nivel de $5 \%$.

As alterações do perfil de ácidos graxos pela formação de isômeros trans (Figuras 3, 4 e 5) em detrimento aos ácidos graxos poliinsaturados (Figuras 1 e 2), demonstraram que a partir de 10 horas de fritura, o OS (Tabela 1) formou um total de 5,4\% de ácidos graxos trans $(2,1 \%$ de isômeros mono trans e $3,3 \%$ de poli trans). Após 50 horas de fritura pode-se observar a formação de $17,1 \%$ de ácidos graxos trans, a qual também foi atribuída ao comprometimento dos ácidos graxos poliinsaturados. No entanto, a GPHS (Tabela 2) apresentou um total de ácidos graxos trans de 28,9\% (20,2\% de ácidos graxos mono trans e $8,7 \%$ de poli trans) antes de ser submetida à fritura, e após 50 horas de processamento apresentou uma concentração de 33,9\% em decorrência à diminuição dos ácidos graxos essenciais das séries $\omega 6$ e $\omega 3$, que passou de $12,8 \%$ no tempo 0 para $7,3 \%$. Assim, a GPHS no final das 50 horas de fritura, continha a mais alta concentração de ácidos graxos trans, a mais baixa concentração de ácidos graxos poliinsaturados e concentração similar de ácidos graxos saturados ao OS (25,6\% e $23,3 \%$, respectivamente). 


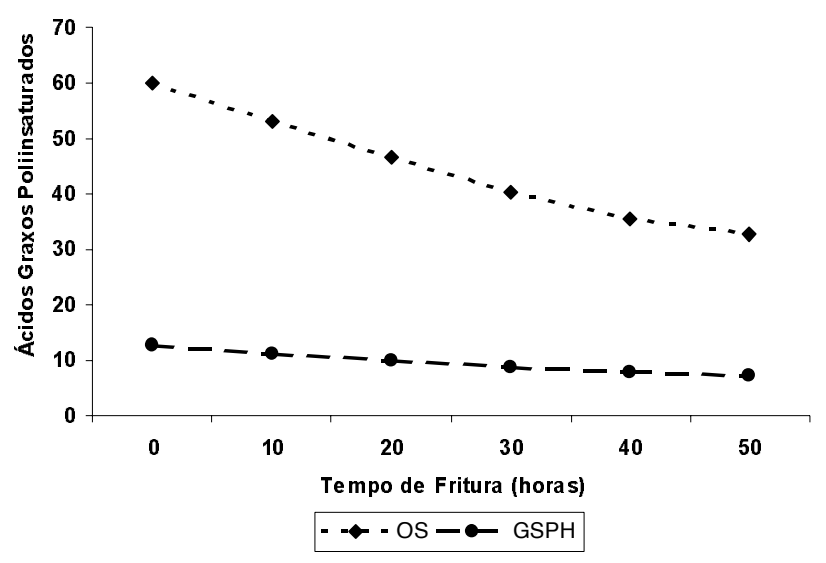

FIGURA 1. Concentrações de ácidos graxos poliinsaturados do OS e da GPHS durante o processo de fritura.

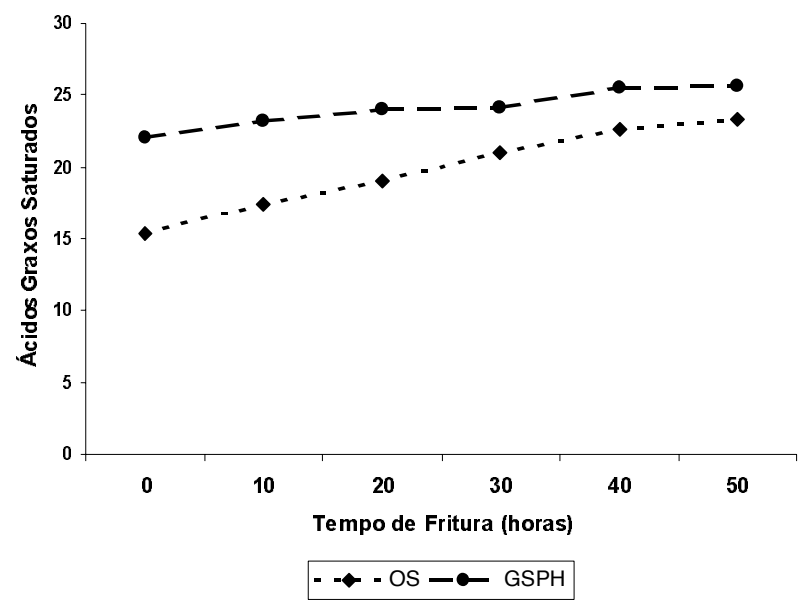

FIGURA 2. Concentrações de ácidos graxos saturados no OS e na GPHS nos diversos tempos de fritura.

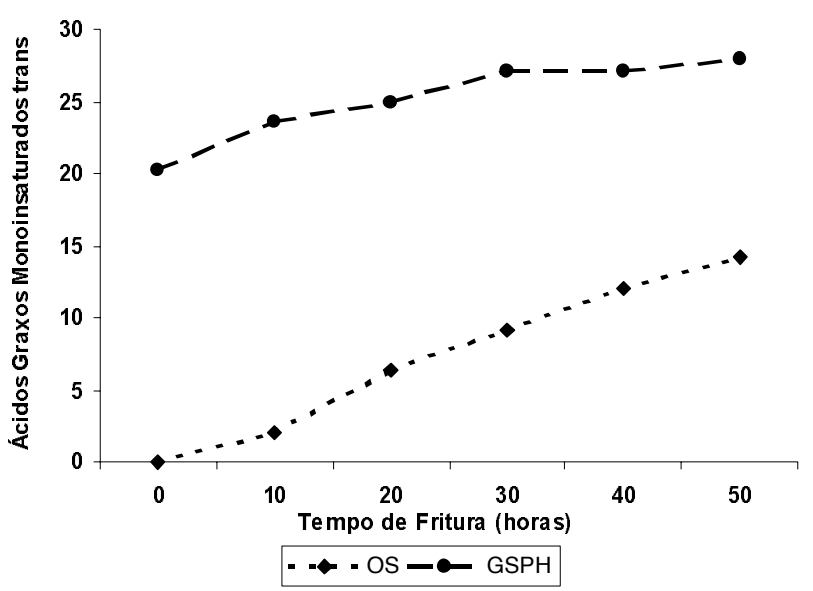

FIGURA 3. Concentrações de ácidos graxos monoinsaturados trans no OS e na GPHS nos diversos tempos de fritura.

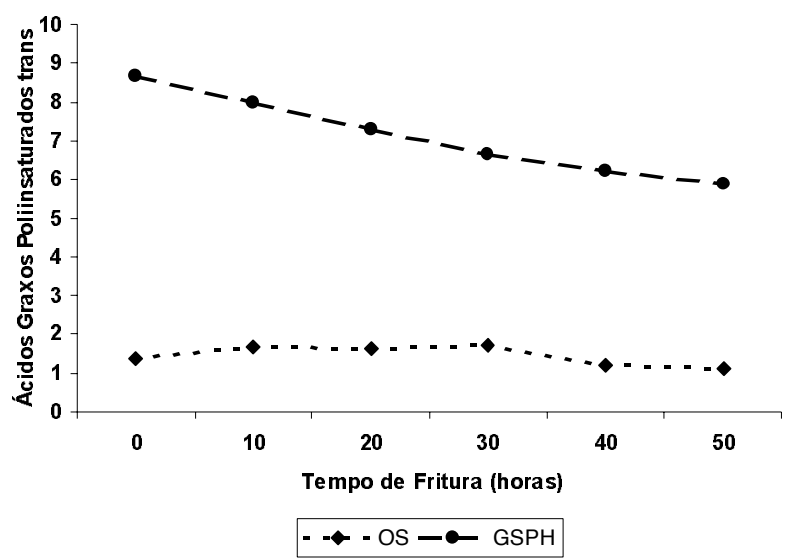

FIGURA 4. Concentrações dos ácidos graxos poliinsaturados trans no OS e na GPHS nos diversos tempos de fritura.

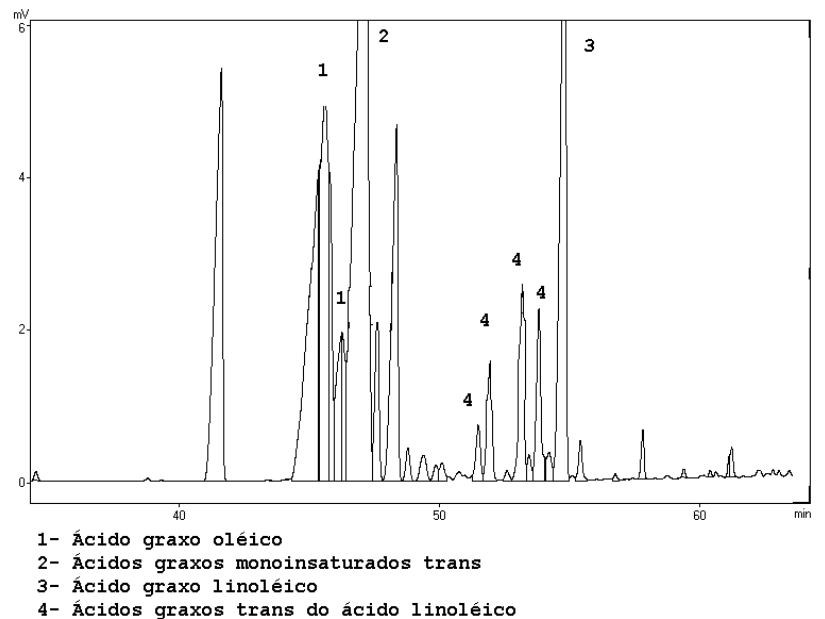

FIGURA 5. Cromatograma ampliado para identificação dos isômeros trans dos ácidos graxos oléico e linoléico formados na GPHS em $10 \mathrm{~h}$ de fritura.

A GPHS sofreu as mesmas modificações do perfil de ácidos graxos em relação ao OS, porém em menores proporções, mostrando uma melhor estabilidade oxidativa durante a fritura de imersão decorrente da sua composição de ácidos graxos. Esse resultado está de acordo com os resultados de XU et al. [18].

Em conformidade com o estudo de WARNER \& MOUNTS [20], os resultados obtidos neste trabalho demonstraram que o aquecimento tanto do OS como da GPHS no processo de fritura, implicaram na diminuição das concentrações dos ácidos graxos essenciais, linoléico, $\alpha$-linolênico e um conseqüente aumento proporcional na concentração dos ácidos graxos saturados.

Assim, também, como encontrado por ROMERO, CUESTA \& SANCHEZ-MUNIZ [13], os ácidos graxos poliinsaturados trans estão presentes nos óleos de fritura e são formados através da isomerização dos ácidos linoléico e linolênico. Deve-se destacar os resultados obtidos com o OS pois este, inicialmente, apresentava apenas $2,1 \%$ de ácidos graxos trans, possivelmente for- 
mado durante o processo de refino. No entanto, com a utilização deste óleo, pode-se observar um aumento no conteúdo dos ácidos graxos trans, chegando a um total de 17,1\%, após 50 horas de fritura (Tabela 1).

Com a GPHS, pode-se observar também um aumento na concentração de ácidos graxos trans (Tabela 2), porém não tão expressivo como o OS.

A concentração de ácido graxo monoinsaturado trans (Tabelas 1 e 2), especificamente o ácido elaídico (C18:1t), foi a maior entre os isômeros trans formados durante o processo de fritura, tanto no OS como na GPHS. Os isômeros trans dos ácidos graxos insaturados, ocorrem no processo de desodorização, assim foi detectado na GPHS, a presença dos isômeros trans dos ácidos oléico, linoléico e $\alpha$-linolênico em maior concentração os isômeros trans monoenóicos, mesmo antes do início do processamento de fritura (Tabela 2).

\section{4 - CONCLUSÕES}

A utilização de óleo de soja é uma alternativa válida como meio de fritura, em relação à gordura parcialmente hidrogenada de soja, considerando seus fatores positivos frente ao custo, que possibilita o seu descarte em menor tempo de utilização e apresenta menor formação de isômeros trans.

\section{5 - REFERÊNCIAS BIBLIOGRÁFICAS}

[1] ASCHERIO, A.; WILLETT, W.C. Health effects of trans fatty acids. Am. J. Clin. Nutr., Bethesda, v. 66, n. 4, p. 1006-1010, 1997.

[2] BARRERA-ARELLANO, D.; BLOCK, J.M. Ácidos grasos trans en aceites hidrogenados: implicaciones técnicas y nutricionales. Grasas y Aceites, Sevilla, v. 44, n. 45, p. 286-293, 1993.

[3] FDA. U.S. Food and Drug Administration. Trans fatty acid in nutrition labeling, nutrient content claims, and health claims. www.cfsan.fda.gov/label.html. Acesso em 12/09/2001.

[4] HARTMAN, L.; LAGO, R.C.A. Rapid preparation of fatty acids methyl esters. Lab. Pract., London, v. 22, p. 475-476, 1973.

[5] KINSELlA, J.E.; BRUCKNER, G.; MAI, J.; SHIMP J. Metabolism of trans fatty acids with emphasis on the effects of trans,trans-octadecadienoate on lipid composition, essential fatty acid, and prostaglandins: an overview. Am. J. Clin. Nutr., Bethesda, v. 34, p. 2307-2318, 1981.

[6] LiCHTENSTEIN, A.H.; JAUHIAINEN, M.; McGLADDERY, S.; AUSMAN, L.M.; JALBERT, S.M.; VILELLA-BACH, M.; EHNHOLM, C.; FROHLICH, J.; SCHAEFER, E.J. Impact of hydrogenated fat on high density lipoprotein subfractions and metabolism, Journal of Lipid Research, Bethesda, v. 42, p. 597-604, 2001.

[7] MANTZIORIS, E.; JAMES, M.J.; GIBSON, R.A.; CLELAND, L.G. Dietary substitution with a-linolenic acid-rich vegetable oil increases eicosapentaenoic acid concentrations. Am. J. Clin. Nutr., Bethesda, v.59, p.1304-1309, 1994.

[8] NAWAR, W.W. Lipids. In: FENNEMA, O.R. Food chemistry. 3.ed. New York: Marcel Dekker, 1996. p
225-319. (Food science and technology: a series of monographs, textbooks, and reference books, 76).

[9] O'BRIEN, R.D. Fats and oils: formulating and processing for applications. Lancaster: Technomic Publishing, 1998. p. 385-410.

[10] OVESEN, L.; LETH, T.; HANSEN, K. Fatty acid composition and contents of trans monounsaturated fatty acids in frying fats, and in margarines and shortenings marketed in Denmark. J. A.m. Oil Chem. Soc., Champaign, v. 75, n. 9, p. 1079-1083, 1998.

[11] POZO-DÍEZ, R.M. Estudo del processo de fritura de alimentos frescos y congelados prefritos. Comportamiento del aceite de semilla de girasol de alto contenido en ácido oleico. Ph D Thesis. Universidad de Alcalá de Henares (Spain) 1995. Apud: ROMERO, A.; CUESTA, C.; SÁNCHEZ-MUNIZ, F.J. Trans fatty acid production in deep fat frying of frozen foods with different oils and frying modalities. Nutr. Res., New York, v. 20, n. 4, p. 599-608, 2000.

[12] RATNAYAKE, W.M.N.; HOLLYWOOD, R., O'GRADY, E., BEARE-ROGERS, J.L. Determination of cis and trans-octadecenoic acids in margarines by gas chromatography-infrared spectrophotometry. J. Am. Oil Chem. Soc., Champaign, v. 67, n. 11, p. 804-810, 1990.

[13] ROMERO, A.; CUESTA, C.; SÁNCHEZ-MUNIZ, F.J. Trans fatty acid production in deep fat frying of frozen foods with different oils and frying modalities. Nutr. Res., New York, v. 20, n. 4, p. 599-608, 2000.

[14] SEBEDIO, J.L.; CATTE, M.; BOUdIER M.A., PREVOST, J., GRANDGIRARD, A. Formation of fatty acid geometrical isomers and of cyclic fatty acid monomers during the finish frying of frozen prefried potatoes. Food Res. Int., Barking, v. 29, n. 2, p. 109-116, 1996.

[15] SUNDRAM, K.; ISMAIL, A.; HAYES, K.C.; JEYAMALAR, R.; PATHMANATHAN, R. Trans (elaidic) fatty acids adversely affect the lipoprotein profile relative to specific saturated fatty acids in human. J. Nutr., Philadelphia, v. 27, n. 3, p. 514, 1997.

[16] TYAGI, V.K.; VASISHTHA, A.K. Changes in the characteristics and composition of oils during deepfat frying. J. Am. Oil Chem. Soc., Champaign, v. 73, n. 4, p. 449-506, 1996.

[17] VALENZUELA, A.; MORGADO, N. Trans fatty acid isomers in human health and in the food industry. Biol. Res., Santiago, v. 32, p. 273-287, 1999.

[18] XU, X.-Q., TRAN, V.H., PALMER, M., WHITE, K. \& SALISBURY, P. Chemical and Physical Analyses and Sensory Evalution of Six Deep-Frying Oils, J. Am. Oil Chem. Soc., Champaign, v. 76, n. 9, p. 1091-1099, 1999.

[19] WALKINS, B.A. Trans fatty acids: a health paradox? Food Technol., Chicago, v. 52, n. 3, p. 120, 1998. [Inform]

[20] WARNER, K.; MOUNTS, T.L. Frying stability of soybean and canola oils with modified fatty acid compositions. J. Am. Oil Chem. Soc., Champaign, v. 70, n. 10, p. 983-988, 1993.

\section{6 - AGRADECIMENTOS}

Ao Conselho Nacional de Desenvolvimento Científico e Tecnológico - CNPq, pela bolsa concedida e à Fundação de Amparo à Pesquisa do Estado de São Paulo - FAPESP, pelo auxílio financeiro. 